NOTICE WARNING CONCERNING COPYRIGHT RESTRICTIONS:

The copyright law of the United States (title 17, U.S. Code) governs the making of photocopies or other reproductions of copyrighted material. Any copying of this document without permission of its author may be prohibited by law. 


\title{
Deciding Quantifier-Free Presburger Formulas using Finite Instantiation based on Parameterized Solution Bounds
}

\author{
Sanjit A. Seshia Randal E. Bryant
}

December 2003

CMU-CS-03-210 3

\author{
School of Computer Science \\ Carnegie Mellon University \\ Pittsburgh, PA 15213
}

This research was supported by ARO grant DAAD19-01-1-0485.

The U.S. Government is authorized to reproduce and distribute reprints for Governmental purposes notwithstanding any copyright annotation thereon. The views and conclusions contained in this document are those of the authors, and should not be interpreted as necessarily representing the official policies or endorsements, either expressed or implied, of the Department of Defense or the U.S. Government. 
Keywords: Presburger arithmetic, Boolean satisfiability, separation (difference-bound) constraints, solution bounds, finite instantiation, decision procedures, theorem proving. 


\begin{abstract}
Given a formula $\Phi$ in quantifier-free Presburger arithmetic, it is well known that, if there is a satisfying solution to $\Phi$, there is one whose size, measured in bits, is polynomially bounded in the size of $\Phi$. In this paper, we consider a special class of quantifier-free Presburger formulas in which most linear constraints are separation (difference-bound) constraints, and the non-separation constraints are sparse. This class has been observed to commonly occur in software verification problems. We derive a new solution bound in terms of parameters characterizing the sparseness of linear constraints and the number of non-separation constraints, in addition to traditional measures of formula size. In particular, the number of bits needed per integer variable is linear in the number of non-separation constraints and logarithmic in the number and size of non-zero coefficients in them, but is otherwise independent of the total number of linear constraints in the formula. The derived bound can be used in a decision procedure based on instantiating integer variables over a finite domain and translating the input quantifier-free Presburger formula to an equi-satisfiable Boolean formula, which is then checked using a Boolean satisfiability solver. We present empirical evidence indicating that this method can greatly outperform other decision procedures.
\end{abstract}





\section{Introduction}

Presburger arithmetic [27] is defined as the first-order theory of the structure $\langle\mathbb{N}, 0,1, \leqslant,+\rangle$, where $\mathbb{N}$ denotes the set of natural numbers. The satisfiability problem for Presburger arithmetic is decidable, but of super-exponential worst-case complexity [13]. Fortunately, for many applications, such as in program analysis (e.g., [28]) and hardware verification (e.g., [8]), the quantifier-free fragment suffices.

A formula $\Phi$ in quantifier-free Presburger arithmetic (QFP) is constructed by combining linear constraints with Boolean operators $(\wedge, \vee, \neg)$. Formally, the $i$ th constraint is of the form $\sum_{j=1}^{n} a_{i, j} x_{j} \geq b_{i}$, where the coefficients and the constant terms are integer constants and the variables $x_{1}, x_{2}, \ldots, x_{n}$ are integer-valued ${ }^{1}$. In this paper, we are concerned with the satisfiability problem for QFP, viz., that of finding a valuation of the variables such that $\Phi$ evaluates to true. That this problem is in NP, and hence NP-complete, can be concluded from the result that integer linear programming is in NP $[6,30,17,23]^{2}$

Thus, if there is a satisfying solution to a QFP formula, there is one whose size, measured in bits, is polynomially bounded in the problem size. Problem size is traditionally measured in terms of the parameters $m, n, \log \mu_{A}$, and $\log \mu_{b}$, where $m$ is the total number of constraints in the formula, $n$ is the number of variables, and $\mu_{A}=\max _{(i, j)}\left|a_{i, j}\right|$ and $\mu_{b}=\max _{i}\left|b_{i}\right|$ are upper bounds on the absolute values of coefficients and constant terms respectively.

The above result suggests the following approach to checking the satisfiability of a QFP formula $\Phi$ :

1. Compute the polynomial bound $S$ on solution size.

2. Search for a satisfying solution to $\Phi$ in the bounded space $\left\{0,1, \ldots, 2^{S}-1\right\}^{n}$.

This approach has been successfully applied to highly restricted sub-classes of QFP, such as equality logic [25] and separation logic [9], and is termed as finite instantiation. The basic idea is to translate $\Phi$ to a Boolean formula by encoding each integer variable as a vector of Boolean variables (a "symbolic bit-vector") of length $S$. The resulting Boolean formula is checked using a Boolean satisfiability (SAT) solver. This approach leverages the dramatic advances in SAT solving made in recent years (e.g., $[20,15])$. It is straightforward to extend the approach to additionally handle the theory of uninterpreted functions and equality, by using, e.g., Ackermann's technique of eliminating function applications [1].

However, a naïve implementation of a decision procedure based on finite instantiation fails for QFP formulas encountered in practice. The problem is that the bound on solution size, $S$, is $O\left(\log m+\log \mu_{b}+m\left[\log m+\log \mu_{A}\right]\right)$. In particular, the presence of the $m \log m$ term means that for practical problems involving hundreds of linear constraints, the Boolean formulas generated are likely to be too large to be decided by present-day SAT solvers.

In this paper, we explore the above finite instantiation-based approach to deciding QFP formulas, but with a focus on formulas generated in software verification. It has been observed, by us and others, that QFP formulas from this domain have:

\footnotetext{
${ }^{1}$ While Presburger arithmetic is defined over $\mathbb{N}$, we interpret the variables over $\mathbb{Z}$ as it is general and more suitable for applications. It is straightforward to translate a formula with integer variables to one where variables are interpreted over $\mathbb{N}$, and vice-versa, by adding (linearly many) additional variables or constraints.

${ }^{2}$ The NP-hardness follows from a straightforward encoding of the 3SAT problem as a 0-1 integer linear program.
} 


\begin{tabular}{|c|c|c|}
\hline Project & $\begin{array}{c}\text { Maximum Fraction of } \\
\text { Non-Separation Constraints }\end{array}$ & $\begin{array}{c}\text { Maximum Width of a } \\
\text { Non-Separation Constraint }\end{array}$ \\
\hline Blast & 0.0276 & 6 \\
\hline Magic & 0.0032 & 2 \\
\hline MIT & 0.0087 & 3 \\
\hline WiSA & 0.0054 & 4 \\
\hline
\end{tabular}

Table 1: Linear Arithmetic Constraints in Software Verification are Mostly Separation Constraints. For each software verification project, the maximum fraction of non-separation constraints is shown, as well as the maximum width of a non-separation constraint, where the maximum is taken over all formulas in the set. The Blast formulas were generated from device drivers written in $\mathrm{C}$, the Magic formulas from an implementation of openssl written in $\mathrm{C}$, the MIT formulas from Java programs, and the WiSA formulas were generated in the checking of format string vulnerabilities.

1. Mainly Separation Constraints: Of the $m$ constraints, $m-k$ are separation constraints, where $k \ll m$. Separation constraints, also called difference-bound constraints, are of the form $x_{i}-x_{j} \bowtie b_{t}$ or $x_{i} \bowtie b_{t}$, where $b_{t}$ is an integer constant, and $\bowtie \in\{>, \geq,=,<, \leq\}$.

2. Sparse Structure: The $k$ non-separation constraints are sparse, with at most $w$ variables per constraint, where $w$ is "small". We will refer to $w$ as the width of the constraint.

Pratt [26] observed that most inequalities generated in program verification are separation constraints. More recently, the authors of the theorem prover Simplify observed in the context of the Extended Static Checker for Java (ESC/Java) project that "the inequalities that occur in program checking rarely involve more than two or three terms" [12]. We have performed a study of formulas selected from various recent software verification projects: the Blast project at Berkeley [16], the Magic project at CMU [10], the Wisconsin Safety Analyzer (WiSA) project ${ }^{3}$, and the software upgrade checking project at MIT [19]. The results of this study, indicated in Table 1, support the afore-mentioned observations regarding the "sparse, mostly separation" nature of constraints in QFP formulas. To our knowledge, no previous decision procedure for QFP has attempted to exploit this problem structure.

We make the following novel contributions in this paper:

- We derive bounds on solutions for QFP formulas, not only in terms of the traditional parameters $m, n, \mu_{A}$, and $\mu_{b}$, but also in terms of $k$ and $w$. In particular, we show that the worst-case number of bits required per integer variable is linear in $k$, but only logarithmic in $w$. Unlike previously derived bounds, ours is independent of the total number of constraints $m$.

- We use the derived bounds in a sound and complete decision procedure for QFP based on finite instantiation, and present empirical evidence that our method can greatly outperform other decision procedures.

Related Work. There has been much work on deciding quantifier-free Presburger arithmetic; we present a brief discussion here and refer the reader to a recent survey [14] for more details. Recent techniques fall into three categories:

\footnotetext{
${ }^{3}$ http : //www.cs.wisc. edu/wisa
} 
- The first class comprises procedures targeted towards solving conjunctions of constraints, with disjunctions handled by enumerating terms in a disjunctive normal form (DNF). Examples include the Omega test [28] and solvers based on other integer linear programming techniques. The drawback of these methods is the need to enumerate the potentially exponentially many terms in the DNF representation.

- The second set of methods attempt to remedy this problem by instead relying on modern SAT solving strategies. The approach works as follows. A Boolean abstraction of the QFP formula $\Phi$ is generated by replacing each linear constraint with a corresponding Boolean variable. If the abstraction is unsatisfiable, then so is $\Phi$. If not, the satisfying assignment (model) is checked for consistency with the theory of quantifier-free Presburger arithmetic, using a ground decision procedure for conjunctions of linear constraints. Assignments that are inconsistent are excluded from later consideration by adding a "lemma" to the Boolean abstraction. The process continues until either a consistent assignment is found, or all (exponentially many) assignments have been explored. Examples of decision procedures in this class that have some support for QFP include CVC [2,3] and ICS [11]. These provers employ the Nelson-Oppen architecture for cooperating decision procedures [22], or some variant of it. Note that the original Nelson-Oppen framework was only defined for disjoint theories. In order to exploit the mostly-separation structure of a formula, one approach could be to combine a decision procedure for a theory of separation constraints with one for a theory of non-separation constraints, but this needs an extension of the Nelson-Oppen framework to apply to these non-disjoint theories.

- The final class of methods are based on finite automata theory (e.g., [31, 14]). The basic idea is to construct a finite automaton corresponding to the input QFP formula $\Phi$, such that language accepted by the automaton consists of the binary encodings of satisfying solutions of $\Phi$. According to a recent experimental evaluation with other methods [14], these techniques are better than others at solving formulas with very large coefficients, but do not scale well with the number of variables and constraints. ${ }^{4}$

The approach we present in this paper is distinct from the categories mentioned above. In particular, the following unique features differentiate it from previous methods:

- It is the first finite instantiation method, translating a QFP formula to SAT in a single step. The clear separation between the translation and the SAT solving allows us to leverage future advances in SAT solving far more easily than other SAT-based procedures.

- It is the first technique, to the best of our knowledge, that exploits the structure of formulas commonly encountered in software verification.

Outline of the paper. The rest of this paper is organized as follows. In Section 2, we discuss background material on bounds on satisfying solutions of integer linear programs. An integer linear program (ILP) is a conjunction of linear constraints, and hence is a special kind of QFP formula. The bounds for QFP follow directly from those for ILPs. Our main theoretical results are presented in Sections 3-5. Section 3 gives bounds for ILPs for the case of $k=0$, when all constraints are separation constraints. In Section 4, we compute a bound for ILPs for arbitrary $k$. In Section 5 , we show how our results extend to arbitrary QFP formulas. We report on experimental results in Section 6 , and conclude in Section 7.

\footnotetext{
${ }^{4}$ Note that automata-based techniques can handle full Presburger arithmetic, not just the quantifier-free fragment.
} 


\section{Background}

In this section, we define the integer linear programming problem formally and state the previous results on bounding satisfying solutions of ILPs. A more detailed discussion on the steps outlined in Section 2.1 can be found in reference books on ILP (e.g. [29, 24]). Useful results on determinants used in the paper are reviewed in Appendix B.

\subsection{Preliminaries}

Consider a system of $m$ linear constraints in $n$ integer-valued variables:

$$
A \mathbf{x} \geq b
$$

Here $A$ is an $m \times n$ matrix with integral entries, $b$ is a $m \times 1$ vector of integral entries, and $\mathbf{x}$ is a $n \times 1$ vector of integer-valued variables. A satisfying solution to system (1) is an evaluation of $\mathbf{x}$ that satisfies (1).

In system (1), the entries in $\mathbf{x}$ can be negative. We can constrain the variables to be non-negative by adding a dummy variable $x_{0}$ that refers to the "zero value," replacing each original variable $x_{i}$ by $x_{i}^{\prime}-x_{0}$, and then adjusting the coefficients in the matrix $A$ to get a new constraint matrix $A^{\prime}$ and the following system: ${ }^{5}$

$$
\begin{aligned}
A^{\prime} \mathbf{x}^{\prime} & \geq b \\
\mathbf{x}^{\prime} & \geq 0
\end{aligned}
$$

Here the system has $n^{\prime}=n+1$ variables, and $\mathbf{x}^{\prime}=\left[x_{1}^{\prime}, x_{2}^{\prime}, \ldots, x_{n}^{\prime}, x_{0}\right]^{T} . A^{\prime}$ has the structure that $a_{i, j}^{\prime}=a_{i, j}$ for $j=1,2, \ldots, n$ and $a_{i, n+1}^{\prime}=-\sum_{j=1}^{n} a_{i, j}$. Note that the last column of $A^{\prime}$ is a linear combination of the previous $n$ columns. As shown in Proposition 1 in Appendix A, system (1) has a solution if and only if system (2) has one.

Finally, adding surplus variables to the system, we can rewrite system (2) as follows:

$$
\begin{aligned}
A^{\prime \prime} \mathbf{x}^{\prime \prime} & =b \\
\mathbf{x}^{\prime \prime} & \geq 0
\end{aligned}
$$

where $A^{\prime \prime}=\left[A \mid-I_{m}\right]$ is an $m \times\left(n^{\prime}+m\right)$ integer matrix formed by concatenating $A$ with the negation of the $m \times m$ identity matrix $I_{m}$.

For convenience we will drop the primes, referring to $A^{\prime \prime}$ and $\mathbf{x}^{\prime \prime}$ simply as $\mathbf{A}$ and $\mathbf{x}$. Rewriting system (3) thus, we get

$$
\begin{aligned}
A \mathbf{x} & =b \\
\mathbf{x} & \geq 0
\end{aligned}
$$

Hereafter we will use the definition in (4). Let $\mu_{A}=\max _{(i, j)}\left|a_{i, j}\right|$ and $\mu_{b}=\max _{t}\left|b_{t}\right|$ be upper bounds on the absolute values of entries of $A$ and $b$ respectively.

\footnotetext{
${ }^{5}$ Note that this procedure can increase the width of a constraint by 1 . The statistics in Table 1 shows the width before this procedure is applied, computed from constraints as they appear in the original formulas.
} 


\subsection{Previous Results}

The results of this paper build on results obtained by Borosh, Treybig, and Flahive [6, 5] on bounding the solution of systems of the form (4). We state their result in the following theorem:

Theorem 1 Consider the augmented matrix $[A \mid b]$ of dimension $m \times\left(n^{\prime}+m+1\right)$. Let $\Delta$ be the maximum of the absolute values of all minors of this augmented matrix. Then, the system (4) has a satisfying solution if and only if it has one with all entries bounded by $(n+2) \Delta$.

However, note that the determinant of a matrix can be more than exponential in the dimension of the matrix [7]. In the case of the Borosh-Flahive-Treybig result, it means that $\Delta$ can be as large as $\frac{\mu^{m}(m+1)^{(m+1) / 2}}{2^{m}}$, where $\mu=\max \left(\mu_{A}, \mu_{b}\right)$.

Papadimitriou $[23,24]$ also gives a bound of similar size, stated in the following theorem:

Theorem 2 If the ILP of (4) has a satisfying solution, then it has a satisfying solution where all entries in the solution vector are bounded by $\left(n^{\prime}+m\right)\left(1+\mu_{b}\right)\left(m \mu_{A}\right)^{2 m+3}$.

Papadimitriou's bound implies that we need $O\left(\log m+\log \mu_{b}+m\left[\log m+\log \mu_{A}\right]\right)$ bits to encode each variable (assuming $\left.n^{\prime}=O(m)\right)$. The Borosh-Flahive-Treybig bound implies needing $O(m[\log m+$ $\log \mu]$ ) bits per variable, which is of the same order.

\section{Bounds for a System of Separation Constraints}

Let us first consider computing solution bounds for an ILP for the case where $k=0$, i.e., system (4) comprises only of separation constraints.

In this case, the left-hand side of each equation comprises exactly three variables: two variables $x_{i}$ and $x_{j}$ where $0 \leq i, j \leq n$ and one surplus variable $x_{l}$ where $n+1 \leq l \leq n+m$. The $t^{\text {th }}$ equation in the system is of the form $x_{i}-x_{j}-x_{l}=b_{t}$.

As we noted in Section 2.1, the matrix $A$ can be written as $\left[A_{o} \mid-I_{m}\right]$ where $A_{o}$ comprises the first $n^{\prime}=n+1$ columns, and $I_{m}$ is the $m \times m$ identity matrix.

The important property of $A_{o}$ is that each row has exactly one +1 entry and exactly one -1 entry, with all other entries 0 . Thus, $A_{o}^{T}$ can be interpreted as the node-arc incidence matrix of a directed graph. Therefore, $A_{o}^{T}$ is totally unimodular (TUM), i.e., every square submatrix of $A_{o}^{T}$ has determinant in $\{0,-1,+1\}$ [24]. Therefore, $A_{o}$ is TUM, and so is $A=\left[A_{o} \mid-I_{m}\right]$.

Now, let us consider using the Borosh-Flahive-Treybig bound stated in Theorem 1. This bound is stated in terms of the minors of the matrix $[A \mid b]$. For the special case of this section, we have the following bound on the size of any minor:

Theorem 3 The absolute value of any minor of $[A \mid b]$ is bounded above by $s \mu_{b}$, where $s=\min (n+$ $1, m)$.

Proof:

Consider any minor $M$ of $[A \mid b]$. Let $r$ be the order of $M$. 
If the minor is obtained by deleting the last column (corresponding to $b$ ), then it is a minor of $A$, and its value is in $\{0,-1,+1\}$ since $A$ is TUM. Thus, the bound of $s \mu_{b}$ is attained for any non-trivial minor with $s \geq 1$ and $\mu_{b} \geq 1$.

Suppose the $b$ column is not deleted.

First, note that the matrix $A$ is of the form $\left[A_{o} \mid-I_{m}\right]$ where the rank of $A_{o}$ is at most $s^{\prime}=\min (n, m)$. This is because $A_{o}$ has dimensions $m \times n+1$, and the last column of $A_{o}$, corresponding to the variable $x_{0}$, is a linear combination of the previous $n$ columns. ${ }^{6}$

Next, suppose the sub-matrix corresponding to $M$ comprises $p$ columns from the $-I_{m}$ part, $r-p-1$ columns from the $A_{o}$ part, and the one column corresponding to $b$. Since permuting the rows and columns of $M$ does not change its absolute value, we can permute the rows of $M$ and the columns corresponding to the $-I_{m}$ part to get the corresponding sub-matrix in the following form:

$$
\left[\begin{array}{c|cccc|c} 
& 0 & \ldots & 0 & -1 & b_{t_{1}} \\
A_{o} & 0 & \ldots & -1 & 0 & b_{t_{2}} \\
\text { part } & \vdots & \ldots & \vdots & \vdots & \vdots \\
& -1 & \ldots & 0 & 0 & b_{t_{p}} \\
& 0 & \ldots & 0 & 0 & b_{t_{p+1}} \\
& \vdots & \ldots & \vdots & \vdots & \vdots \\
& 0 & \ldots & 0 & 0 & b_{t_{r}}
\end{array}\right]
$$

Expanding $M$ along the last column, we get

$$
|M|=\left|b_{t_{1}} M_{1}-b_{t_{2}} M_{2}+b_{t_{3}} M_{3}-\ldots(-1)^{r-1} b_{t_{r}} M_{r}\right|
$$

where each $M_{i}$ is a minor corresponding to a submatrix of $A$.

However, notice that $M_{i}=0$ for all $1 \leq i \leq p$, since each of those minors have an entire column (from the $-I_{m}$ part) equal to 0 . Therefore, we can reduce the right-hand side to the sum of $r-p$ terms:

$$
|M| \leq\left|b_{t_{p+1}} M_{p+1}\right|+\left|b_{t_{p+2}} M_{p+2}\right|+\ldots\left|b_{t_{r}} M_{r}\right|
$$

Notice that, so far, we have not made use of the special structure of $A$.

Now, observing that $A$ is TUM, $\left|M_{i}\right| \leq 1$ for all $i$.

$$
|M| \leq\left|b_{t_{p+1}}\right|+\left|b_{t_{p+2}}\right|+\ldots+\left|b_{t_{r}}\right|
$$

For all $i,\left|b_{t_{i}}\right| \leq \mu_{b}$. Further, since each non-zero $M_{i}$ can be of order at most $s^{\prime}, r-p \leq s=$ $\min \left(s^{\prime}+1, m\right) \cdot{ }^{7}$ Therefore, we get

$$
|M| \leq s \mu_{b}
$$

Using the terminology of Theorem 1 , we have $\Delta \leq s \mu_{b}$. Thus, the bound in this case is $(n+2) s \mu_{b}$. Thus, $S$, the bound on the number of bits per variable, is

$$
\left\lceil\log (n+2)+\log s+\log \mu_{b}\right\rceil
$$

\footnotetext{
${ }^{6}$ Refer to the construction of system (2) from system (1).

${ }^{7}$ We use $s^{\prime}+1$ and not $s^{\prime}$ to account for the case where $p=0$. The minimum with $m$ is taken because $s^{\prime}+1$ can exceed $m$ but $b$ has only $m$ elements.
} 
Formulas generated from verification problems tend to be overconstrained, so we assume $n<m$. Thus, $s=n+1$, and the bound reduces to $O\left(\log n+\log \mu_{b}\right)$ bits per variable.

Remark. The only property of the $A$ matrix that the proof of Theorem 3 relies on is the totally unimodular (TUM) property. Thus, Theorem 3 would also apply to any system of linear constraints whose coefficient matrix is TUM. Examples of such matrices include interval matrices, or more generally network matrices. Note that the TUM property can be tested for in polynomial time [29].

\section{Bounds for a Sparse System of Mainly Separation Constraints}

We now consider the general case for ILPs, where we have $k$ non-separation constraints, each referring to at most $w$ variables.

Without loss of generality, we can reorder the rows of matrix $A$ so that the $k$ non-separation constraints are the top $k$ rows, and the separation constraints are the bottom $m-k$ rows. Reordering the rows of $A$ can only change the sign of any minor of $[A \mid b]$, not the absolute value. Thus, the matrix $[A \mid b]$ can be put into the following form:

$$
\left[\begin{array}{c|l|c}
A_{1} & -b_{1} \\
& -I_{2} & b_{2} \\
& & \vdots \\
& b_{m}
\end{array}\right]
$$

Here, $A_{1}$ is a $k \times n+1$ dimensional matrix corresponding to the non-separation constraints, $A_{2}$ is a $m-k \times n+1$ dimensional matrix with the separation constraints, $I_{m}$ is the $m \times m$ identity corresponding to the surplus variables, and the last column is the vector $b$.

The matrix comprised of $A_{1}$ and $A_{2}$ will be referred to, as before, as $A_{o}$. Note that each row of $A_{1}$ has at most $w$ non-zero entries, and each row of $A_{2}$ has exactly one +1 and one -1 with the remaining entries 0 . Thus, $A_{2}$ is TUM.

We prove the following theorem:

Theorem 4 The absolute value of any minor of $[A \mid b]$ is bounded above by $s \mu_{b}\left(\mu_{A} w\right)^{k}$, where $s=\min (n+1, m)$.

Proof:

Consider any minor $M$ of $[A \mid b]$, and let $r$ be its order.

As in Theorem 3, if $M$ includes $p$ columns from the $-I_{m}$ part of $A$, then we can infer that $r-p \leq s$. (Our proof of this property in Theorem 3 made no assumptions on the form of $A_{o}$.)

If $M$ includes the last column $b$, then as in the proof of Theorem 3, we can conclude that

$$
|M| \leq(r-p) \mu_{b}\left[\max _{j=1}^{r}\left|M_{j}\right|\right]
$$

where $M_{j}$ is a minor of $A_{o}$.

If $M$ does not include $b$, then it is a minor of $A$. Without loss of generality, we can assume that $M$ does not include a column from the $-I_{m}$ part of $A$, since such columns only contribute to the sign of the determinant. 
So, let us consider bounding a minor $M_{j}$ of $A_{o}$ of order $r$ ( or $r-1$, if $M$ includes the $b$ column). Since $A_{o}=\left[\frac{A_{1}}{A_{2}}\right]$, consider expanding $M_{j}$, using the standard determinant expansion by minors along the top $k$ rows corresponding to non-separation constraints (see Equation 8 in Appendix B). Each term in the expansion is (up to a sign) the product of at most $k$ entries from the $A_{1}$ portion, one from each row, and a minor from $A_{2}$. Since $A_{2}$ is TUM, each product term is bounded in absolute value by $\mu_{A}^{k}$. Furthermore, there can be at most $w^{k}$ non-zero terms in the expansion, since each non-zero product term is obtained by choosing one non-zero element from each of the rows of the $A_{1}$ portion of $M_{j}$, and this can be done in at most $w^{k}$ ways.

Therefore, $\left|M_{j}\right|$ is bounded by $\left(\mu_{A} w\right)^{k}$. Combining this with the inequality (5), and since $r-p \leq s$, we get

$$
|M| \leq s \mu_{b}\left(\mu_{A} w\right)^{k}
$$

which is what we set out to prove.

Thus, we conclude that $\Delta \leq s \mu_{b}\left(\mu_{A} w\right)^{k}$, where $s=\min (n+1, m)$. From Theorems 1 and 4 , the solution bound is $(n+2) \Delta$. Thus, $S$ is

$$
\left\lceil\log (n+2)+\log s+\log \mu_{b}+k\left(\log \mu_{A}+\log w\right)\right\rceil
$$

We make the following observations about the bound derived above, assuming as before, that $n<m$, and so $s=n+1$ :

- Dependence on Parameters: We observe that the bound is linear in $k$, logarithmic in $\mu_{A}, w$, $n$, and $\mu_{b}$. In particular, the bound is independent of the total number of linear constraints, $m$.

- Worst-case Asymptotic Growth: In the worst case, $k=m, w=n+1$, and $n=O(m)$, and we get the $O\left(\log m+\log \mu_{b}+m\left[\log m+\log \mu_{A}\right]\right)$ bound of Papadimitriou.

- Typical-case Asymptotic Growth: As observed in Section 1, w is typically a small constant, so the number of bits needed per variable is $O\left(\log n+\log \mu_{b}+k \log \mu_{A}+k\right)$. In many cases, $\mu_{A}$ is also a small constant, simplifying the bound to $O\left(\log n+\log \mu_{b}+k\right)$ bits per variable.

- Representing Non-separation Constraints: There are many ways to represent non-separation constraints and these have an impact on the bound we derive. In particular, it is possible to transform a system of non-separation constraints to one with at most three variables per constraint. For example, the linear constraint $x_{1}+x_{2}+x_{3}+x_{4}=x_{5}$ can be rewritten as:

$$
\begin{aligned}
& x_{1}+x_{1}^{\prime}=x_{5} \\
& x_{2}+x_{2}^{\prime}=x_{1}^{\prime} \\
& x_{3}+x_{4}=x_{2}^{\prime}
\end{aligned}
$$

For the original representation, $k=1$ and $w=5$, while for the new representation $k=3$ and $w=3$. Since our bound is linear in $k$ and logarithmic in $w$, the original representation would yield a tighter bound.

Similarly, one can eliminate variables with coefficients greater than 1 in absolute value by a similar process of adding new non-separation constraints. Again, since the bound is logarithmic in $\mu_{A}$, it would be preferable to avoid adding new non-separation constraints. 
The derived bound only yields benefits in the case when the system has few non-separation constraints which themselves are sparse. In this case, we can instantiate variables over a finite domain that is much smaller than that obtained without making any assumptions on the structure of the system.

\section{Bounds for Arbitrary Quantifier-Free Presburger Formulas}

We now return to the original goal of this paper, that of finding a solution bound for an arbitrary QFP formula $\Phi$. Suppose that $\Phi$ has $m$ linear constraints $\phi_{1}, \phi_{2}, \ldots, \phi_{m}$, of which $m-k$ are separation constraints, and $n$ variables $x_{1}, x_{2}, \ldots, x_{n}$. As before, we assume that each non-separation constraint has at most $w$ variables, $\mu_{A}$ is the maximum over the absolute values of coefficients $a_{i, j}$ of variables, and $\mu_{b}$ is the maximum over the absolute values of constants $b_{i}$ appearing in the constraints.

We prove the following theorem.

Theorem 5 If $\Phi$ is satisfiable, there is a solution to $\Phi$ that is bounded by $(n+2) \Delta$ where

$$
\Delta=s\left(\mu_{b}+1\right)\left(\mu_{A} w\right)^{k}
$$

and $s=\min (n+1, m)$.

Proof: Let $\sigma$ be a (concrete) model of $\Phi$. Let $m^{\prime}$ constraints, $\phi_{i_{1}}, \phi_{i_{2}}, \ldots, \phi_{i_{m^{\prime}}}$, evaluate to true under $\sigma$, the rest evaluating to false. Let $A^{\prime}=\left[a_{i, j}\right]$ be a $m^{\prime} \times n$ matrix in which each row comprises the coefficients of variables $x_{1}, x_{2}, \ldots, x_{n}$ in a constraint $\phi_{i_{k}}, 1 \leq k \leq m^{\prime}$. Thus, $A^{\prime}=\left[a_{i, j}\right]$ where $i \in\left\{i_{1}, \ldots, i_{m^{\prime}}\right\}$.

Now consider a constraint $\phi_{i_{k}}$ where $k>m^{\prime}$, that evaluates to false under $\sigma . \phi_{i_{k}}$ is the inequality

$$
\sum_{j=1}^{n} a_{i_{k}, j} x_{j} \geq b_{i_{k}}
$$

Then $\sigma$ satisfies $\neg \phi_{i_{k}}$ which is the inequality

$$
\sum_{j=1}^{n} a_{i_{k}, j} x_{j}<b_{i_{k}}
$$

or equivalently,

$$
\sum_{j=1}^{n}-a_{i_{k}, j} x_{j} \geq-b_{i_{k}}+1
$$

Let $A^{\prime \prime}$ be a $\left(m-m^{\prime}\right) \times n$ matrix corresponding to the coefficients of variables in constraints $\neg \phi_{i_{m^{\prime}+1}}$, $\neg \phi_{i_{m^{\prime}+2}}, \ldots, \neg \phi_{i_{m}}$. Thus, $A^{\prime \prime}=\left[-a_{i, j}\right]$ where $i \in\left\{i_{m^{\prime}+1}, \ldots, i_{m}\right\}$.

Finally, let $b=\left[b_{i_{1}}, b_{i_{2}}, \ldots, b_{i_{m^{\prime}}},-b_{i_{m^{\prime}+1}}+1,-b_{i_{m^{\prime}+2}}+1, \ldots,-b_{i_{m}}+1\right]^{T}$

Clearly, $\sigma$ is a satisfying solution to the ILP given by

$$
\left[\frac{A^{\prime}}{A^{\prime \prime}}\right] \mathbf{x} \geq b
$$


Also, if the system (6) has a satisfying solution then $\Phi$ is satisfied by that solution. Thus, $\Phi$ and the system (6) are equi-satisfiable, for every possible system (6) we construct in the manner described above.

By Theorems 1 and 4, we can conclude that if system (6) has a satisfying solution, it has one bounded by $(n+2) \Delta$ where

$$
\Delta=s\left(\mu_{b}+1\right)\left(\mu_{A} w\right)^{k}
$$

and $s=\min (n+1, m)$. Moreover, this bound works for every possible system (6).

Therefore, if $\Phi$ has a satisfying solution, it has one bounded by $(n+2) \Delta$.

Thus, to generate the Boolean encoding of the starting QFP formula, we must encode each integer variable as a symbolic bit-vector of length $S=\lceil\log [(n+2) \Delta]\rceil=\left\lceil\log (n+2)+\log s+\log \left(\mu_{b}+1\right)+\right.$ $\left.k\left(\log \mu_{A}+\log w\right)\right\rceil$.

Remark. In the preceding discussion, we have used a single bit-vector length for all integer variables appearing in the formula $\Phi$. This is conservative. In general, we can partition the set of variables into classes such that two variables are placed in the same class if there is a constraint in which they both appear with non-zero coefficients. For each class, we separately compute parameters $n, k, \mu_{b}, \mu_{A}$, and $w$, resulting in a separately computed bit-vector length for each class. The correctness of this partitioning optimization follows from a reduction to ILP as performed in the proof of Theorem 5 , and the observation that a satisfying solution to a system of ILPs, no two of which share a variable, can be obtained by solving them independently and concatenating the solutions.

\section{Implementation and Experimental Results}

We used the bound derived in the previous section to implement a decision procedure based on finite instantiation. Integer variables in the QFP formula are encoded as symbolic bit-vectors large enough to express any integer value within the bound. Arithmetic operators are implemented as arbitrary-precision bit-vector arithmetic operations. Equalities and inequalities over integer expressions are translated to corresponding relations over bit-vector expressions. The resulting Boolean formula is passed as input to a SAT solver.

We implemented our procedure as part of $\mathrm{UCLID}^{8}$, which is written in Moscow ML ${ }^{9}$. In our implementation we used the $\mathrm{zChaff}$ SAT solver ${ }^{10}$, version 2003.7.22. We compared UCLID's performance with that of the SAT-based prover ICS (the latest version 2.0) ${ }^{11}$ and the automata-based procedure $\mathrm{LASH}^{12}$. While LASH is sound and complete for QFP, ICS 2.0 is incomplete; i.e., it can report a formula to be satisfiable when it is not. The ground decision procedure ICS uses is the Simplex linear programming algorithm with some additional heuristics to deal with integer variables. However, in our experiments, both UCLID and ICS returned the same answer whenever they both terminated within the timeout. ${ }^{13}$

\footnotetext{
${ }^{8}$ http://www.cs.cmu.edu/ ${ }^{\sim}$ uclid

${ }^{9}$ http: //www.dina.dk/ sestoft/mosml.html

${ }^{10}$ http://ee.princeton.edu/ chaff/zchaff .php

${ }^{11}$ http: //www. icansolve.com

${ }^{12} \mathrm{http}: / /$ www .montefiore.ulg.ac.be/ boigelot/research/lash

${ }^{13} \mathrm{We}$ also attempted comparisons with CVC-Lite (the new version of CVC which includes a ground decision procedure for QFP [3]). However, the implementation was too unstable to be able to make useful comparisons. We intend to perform a comparative evaluation when a stable implementation become available.
} 
For benchmarks, we used several formulas from the Wisconsin Safety Analyzer project on checking format string vulnerabilities. The benchmarks include both satisfiable and unsatisfiable formulas in an extension of QFP with uninterpreted functions. Uninterpreted functions were first eliminated using Ackermann's technique [1], and the decision procedures were run on the resulting QFP formula. Some characteristics of the formulas are displayed in Table 2. For each formula, we indicate whether it is satisfiable or not, and also give the values of parameters $n, m, k, w, \mu_{A}$ and $\mu_{b}$ corresponding to the variable class for which $S=[\log [(n+2) \Delta]\rceil$ is largest, i.e, for which we need the largest number of bits per variable. Note that the total numbers of variables and constraints, for all variable classes, are larger: For example, for the benchmark xs-30-40, the formula has 115 variables and 2610 constraints in all. The formulas involve the combination of linear constraints by arbitrary Boolean operators $(\wedge, \vee, \neg)$. The key characteristics of formulas generated in this class of problems is that they vary in $n, m$, and $\mu_{b}$, but the values of $k, w$, and $\mu_{A}$ are fixed at a small value.

Experiments were performed on a Pentium-IV 2 GHz machine with 1 GB of RAM running Linux. A timeout of 900 seconds was imposed on each run.

\begin{tabular}{|c|c|c|c|c|c|c|c|c|c|c|c|c|c|c|}
\hline \multirow[t]{3}{*}{ Formula } & \multirow[t]{3}{*}{ Ans. } & \multicolumn{7}{|c|}{ Max. Parameters } & \multirow{2}{*}{\multicolumn{3}{|c|}{$\begin{array}{l}\text { UCLID Time } \\
\text { (sec.) }\end{array}$}} & \multicolumn{3}{|c|}{ ICS } \\
\hline & & \multirow[t]{2}{*}{$n$} & \multirow[t]{2}{*}{$m$} & \multirow[t]{2}{*}{$k$} & \multirow[t]{2}{*}{$w$} & \multirow[t]{2}{*}{$\mu_{A}$} & \multirow[t]{2}{*}{$\mu_{b}$} & \multirow[t]{2}{*}{$S$} & & & & \multirow{2}{*}{$\begin{array}{l}\# \text { (Inc. } \\
\text { assn.) }\end{array}$} & \multicolumn{2}{|c|}{ Time (sec.) } \\
\hline & & & & & & & & & Enc. & SAT & Total & & Gnd. & Total \\
\hline$s-20-20$ & $\overline{\text { SAT }}$ & 28 & 437 & 6 & 5 & 4 & 21 & 41 & 8.98 & 5.86 & 14.84 & 904 & 23.32 & 23.76 \\
\hline $\mathrm{s}-20-30$ & SAT & 28 & 437 & 6 & 5 & 4 & 30 & 41 & 9.02 & 26.01 & 35.03 & 1887 & 51.68 & 52.29 \\
\hline$s-20-40$ & UNS & 28 & 437 & 6 & 5 & 4 & 40 & 41 & 9.18 & 363.70 & 372.88 & 25776 & 618.59 & 633.58 \\
\hline $\mathrm{s}-30-30$ & SAT & 38 & 792 & 6 & 5 & 4 & 31 & 42 & 11.93 & 12.29 & 24.22 & 2286 & 268.21 & 269.42 \\
\hline $\mathrm{s}-30-40$ & SAT & 38 & 792 & 6 & 5 & 4 & 40 & 42 & 12.00 & 54.50 & 66.50 & 7311 & 860.71 & * \\
\hline$x s-20-20$ & SAT & 49 & 668 & 6 & 5 & 4 & 21 & 42 & 10.23 & 13.21 & 23.44 & 2307 & 91.31 & 92.87 \\
\hline$x s-20-30$ & SAT & 49 & 668 & 6 & 5 & 4 & 30 & 43 & 10.48 & 26.64 & 37.12 & 15656 & 765.44 & * \\
\hline$x s-20-40$ & - & 49 & 668 & 6 & 5 & 4 & 40 & 43 & 10.49 & * & $*$ & 20590 & 867.00 & * \\
\hline$x s-30-40$ & SAT & 69 & 1288 & 6 & 5 & 4 & 40 & 44 & 17.71 & 33.68 & 51.39 & 9927 & 890.08 & * \\
\hline
\end{tabular}

Table 2: Benchmark characteristics and experimental results. For UCLID, we list the time taken to decide the formula including a breakup into the encoding time ("Enc.") and the time taken by the SAT solver ("SAT"). For ICS, we give the total time, the number of inconsistent Boolean assignments analyzed by the ground decision procedure ("\#(Inc. assn.)"), as well as the overall time taken by the ground decision procedure ("Gnd."). A "*" indicates that the decision procedure timed out after $900 \mathrm{sec}$. LASH was unable to complete within the timeout on any formula.

A comparison of UCLID versus ICS is displayed in Table 2. LASH was unable to complete on any benchmark within the timeout; we attribute this to the relatively large number of variables and constraints in our formulas, and note that Ganesh et al. obtained similar results in their study [14]. From Table 2, we observe that UCLID outperforms ICS on all benchmarks, terminating within the timeout on several benchmarks on which ICS does not.

The reason for UCLID's superior performance is the formula structure, where $k, w$, and $\mu_{A}$ remain fixed at a low value while $m, n$, and $\mu_{b}$ increase. Thus, the maximum number of bits per variable is only moderately large (about 40 ), even as $m$ increases substantially, and the resulting SAT problem is within the capacity of zChaff. Also, we note that UCLID's run-time is dominated by the SAT time, since the time to compute the parameter values and generate the SAT-encoding is polynomial in the input size.

For ICS, we note that the run-time is dominated by the time taken by the ground decision procedure. 
We observe that the number of inconsistent Boolean assignments alone is not a precise indicator of total run-time, which also depends on the time taken by the ground decision procedure in ruling out a single Boolean assignment.

\section{Conclusions and Future Work}

In this paper, we have presented a formal approach to exploiting the "sparse, mainly separation constraint" nature of quantifier-free Presburger formulas encountered in software verification. Our approach is based on deriving a new parameterized bound on satisfying solutions to QFP formulas. Experimental results show the benefits of using the derived bound in a SAT-based decision procedure based on finite instantiation.

Note that the bounds we have derived and used in our experiments are conservative. First, the size of minors in a particular problem instance might be far smaller than the bounds we have computed. It is unclear how this can be exploited, since there are exponentially many minors in the dimensions of the input matrix. Second, for certain special cases, one can improve the $(n+2) \Delta$ bound. For example, if all the constraints are originally equalities and the system of constraints has full rank, a bound of $\Delta$ suffices [4]. Thirdly, in cases where the value of $\mu_{b}$ is very large due to the presence of a single large constant, one might want to use a less conservative analysis than is performed in the proof of Theorem 4.

In our implementation, we translate a QFP formula to a Boolean formula in a single step. An alternative approach is to perform this transformation lazily, increasing the bit-vector size "on demand". This lazy encoding approach works, in brief, as follows. (Details can be found in [18].) We start with an encoding size for each integer variable that is smaller than that prescribed by the bound. If the resulting Boolean formula is satisfiable, so is the original QFP formula. If not, the proof of unsatisfiability generated by the SAT solver is used to generate a sound abstraction of the original formula, which can be checked with a sound and complete decision procedure for QFP (such as the one proposed in this paper). If this decision procedure concludes that the abstraction is unsatisfiable, so is the original formula, but if not, it provides a counterexample which indicates the necessary increase in the encoding size, and the procedure repeats. The advantage of this lazy approach is twofold: (1) It avoids using the conservative bounds we have derived in this paper, and (2) if the generated abstractions are small, the sound and complete decision procedure used by this approach will run much faster than if it were fed the original formula. The bound $S$ that we derive in this paper implies an upper bound $n S$ on the number of iterations of this lazy encoding procedure; thus the lazy encoding procedure needs only polynomially many iterations before it terminates with the correct answer. Using the decision procedure proposed in this paper with the above lazy encoding approach is an interesting avenue for future work.

Finally, it would also be interesting to explore applications other than software verification that share the "sparse, mainly separation constraints" property.

\section{Acknowledgments}

Joël Ouaknine pointed us to results of Borosh, Flahive, and Treybig on which our results rely. We thank him and K. Subramani for useful discussions and feedback on earlier drafts. We are grateful to Sagar Chaki, Michael Ernst, Vinod Ganapathy, Somesh Jha, Ranjit Jhala, and Stephen 
McCamant for providing us with benchmark formulas, and Leonardo de Moura for help with ICS.

\section{References}

[1] W. Ackermann. Solvable Cases of the Decision Problem. North-Holland, Amsterdam, 1954.

[2] C. Barrett, D. Dill, and A. Stump. Checking satisfiability of first-order formulas by incremental translation to SAT. In E. Brinksma and K. G. Larsen, editors, Proc. 14th Intl. Conference on Computer-Aided Verification (CAV'02), LNCS 2404, pages 236-249. Springer-Verlag, July 2002.

[3] S. Berezin, V. Ganesh, and D. L. Dill. An online proof-producing decision procedure for mixed-integer linear arithmetic. In TACAS'03, LNCS 2619, pages 521-536, 2003.

[4] I. Borosh, M. Flahive, D. Rubin, and L. B. Treybig. A sharp bound for solutions of linear Diophantine equations. Proceedings of the American Mathematical Society, 105(4):844-846, April 1989.

[5] I. Borosh, M. Flahive, and L. B. Treybig. Small solutions of linear Diophantine equations. Discrete Mathematics, 58:215-220, 1986.

[6] I. Borosh and L. B. Treybig. Bounds on positive integral solutions of linear Diophantine equations. Proceedings of the American Mathematical Society, 55(2):299-304, March 1976.

[7] Joel Brenner and Larry Cummings. The Hadamard maximum determinant problem. American Mathematical Monthly, 79:626-630, June-July 1972.

[8] R. Brinkmann and R. Drechsler. RTL-datapath verification using integer linear programming. In Proceedings of the IEEE VLSI Design Conference, pages 741-746, 2002.

[9] R. E. Bryant, S. K. Lahiri, and S. A. Seshia. Modeling and verifying systems using a logic of counter arithmetic with lambda expressions and uninterpreted functions. In E. Brinksma and K. G. Larsen, editors, Proc. Computer-Aided Verification (CAV'02), LNCS 2404, pages 78-92, July 2002 .

[10] Sagar Chaki, Edmund M. Clarke, Alex Groce, Somesh Jha, and Helmut Veith. Modular verification of software components in C. In Proc. 25th International Conference on Software Engineering (ICSE), pages 385-395, 2003.

[11] Leonardo de Moura, Harald Rueß, and Maria Sorea. Lazy theorem proving for bounded model checking over infinite domains. In Proc. 18th International Conference on Automated Deduction (CADE), pages 438-455, 2002.

[12] David Detlefs, Greg Nelson, and James B. Saxe. Simplify: A theorem prover for program checking. Technical Report HPL-2003-148, HP Laboratories Palo Alto, 2003.

[13] M. J. Fischer and M. O. Rabin. Super-exponential complexity of Presburger arithmetic. Proceedings of SIAM-AMS, 7:27-41, 1974.

[14] V. Ganesh, S. Berezin, and D. L. Dill. Deciding Presburger arithmetic by model checking and comparisons with other methods. In Formal Methods in Computer-Aided Design (FMCAD '02), LNCS 2517, pages 171-186. Springer-Verlag, November 2002. 
[15] E. Goldberg and Y. Novikov. BerkMin: A fast and robust SAT solver. In Design Automation and Test in Europe (DATE) 2002, pages 142-149, 2002.

[16] Thomas A. Henzinger, Ranjit Jhala, Rupak Majumdar, and Gregoire Sutre. Lazy abstraction. In Proc. 29th ACM Symposium on Principles of Programming Languages, pages 58-70, 2002.

[17] R. Kannan and C. L. Monma. On the computational complexity of integer programming problems. In Optimisation and Operations Research, volume 157 of Lecture Notes in Economics and Mathematical Systems, pages 161-172. Springer-Verlag, 1978.

[18] Daniel Kroening, Joël Ouaknine, Sanjit Seshia, and Ofer Strichman. Abstraction-based satisfiability solving of Presburger arithmetic. Technical Report CMU-CS-04-100, Computer Science Department, Carnegie Mellon University, 2004.

[19] Stephen McCamant and Michael D. Ernst. Predicting problems caused by component upgrades. In Proceedings of the 11th ACM SIGSOFT Symposium on Foundations of Software Engineering (FSE), pages 287-296, 2003.

[20] M. Moskewicz, C. Madigan, Y. Zhao, L. Zhang, and S. Malik. Chaff: Engineering an efficient SAT solver. In 38th Design Automation Conference (DAC '01), pages 530-535, June 2001.

[21] T. Muir and W. Matzler. A Treatise on the Theory of Determinants. Dover, New York, 1960.

[22] G. Nelson and D. C. Oppen. Simplification by cooperating decision procedures. ACM Transactions on Programming Languages and Systems, 1(2):245-257, 1979.

[23] Christos H. Papadimitriou. On the complexity of integer programming. Journal of the ACM, 28(4):765-768, 1981.

[24] Christos H. Papadimitriou and Kenneth Steiglitz. Combinatorial Optimization: Algorithms and Complexity, chapter 13. Prentice-Hall, 1982.

[25] A. Pnueli, Y. Rodeh, O. Shtrichman, and M. Siegel. Deciding equality formulas by smalldomain instantiations. In N. Halbwachs and D. Peled, editors, Computer-Aided Verification, volume 1633 of Lecture Notes in Computer Science, pages 455-469. Springer-Verlag, July 1999.

[26] Vaughan Pratt. Two easy theories whose combination is hard. Technical report, Massachusetts Institute of Technology, 1977. Cambridge, MA.

[27] M. Preßburger. Über die Vollständigkeit eines gewissen Systems der Arithmetik ganzer Zahlen, in welchem die Addition als einzige Operation hervortritt. Comptes-rendus du Premier Congrès des Mathématiciens des Pays Slaves, 395:92-101, 1929.

[28] William Pugh. The omega test: A fast and practical integer programming algorithm for dependence analysis. In Supercomputing, pages 4-13, 1991.

[29] Alexander Schrijver. Theory of Linear and Integer Programming. John Wiley and Sons, 1998.

[30] J. von zur Gathen and M. Sieveking. A bound on solutions of linear integer equalities and inequalities. Proceedings of the American Mathematical Society, 72(1):155-158, October 1978.

[31] Pierre Wolper and Bernard Boigelot. An automata-theoretic approach to Presburger arithmetic constraints. In Proc. Static Analysis Symposium, LNCS 983, pages 21-32, September 1995. 


\section{A Proof of Transformation to System 2}

Proposition 1 System (1) has a solution if and only if system (2) has one.

Proof: For the "if part", suppose we have a solution $\mathbf{x}^{\prime}$ to (2). Construct a candidate solution vector $\mathbf{x}$ by setting $x_{j}=x_{j}^{\prime}-x_{0}$. Then, consider the $i$ th constraint in $A^{\prime}$, for any $i$. The following sequence of equalities holds:

$$
\begin{aligned}
\left(\sum_{j=1}^{n} a_{i, j}^{\prime} x_{j}^{\prime}\right)+a_{i, n+1}^{\prime} x_{0} & \geq b_{i} \\
\left(\sum_{j=1}^{n} a_{i, j} x_{j}^{\prime}\right)+x_{0}\left(-\sum_{j=1}^{n} a_{i, j}\right) & \geq b_{i} \\
\sum_{j=1}^{n} a_{i, j}\left(x_{j}^{\prime}-x_{0}\right) & \geq b_{i} \\
\sum_{j=1}^{n} a_{i, j} x_{j} & \geq b_{i}
\end{aligned}
$$

Thus, we can conclude that the $i$ th constraint of $A$ is satisfied by $\mathbf{x}$ for all $i$. Thus, we have found a solution to system (1).

Now consider the "only if" part, where we start with a solution to system (1). Clearly, any value of $\mathbf{x}^{\prime}$ that sets $x_{j}^{\prime}=x_{j}+x_{0}$ for all $j$ will satisfy $A^{\prime} \mathbf{x}^{\prime} \geq b$. But we also need to satisfy $\mathbf{x}^{\prime} \geq 0$. If none of the $x_{j}$ are negative, then simply set $x_{j}^{\prime}=x_{j}$ and $x_{0}=0$ and we are done. Otherwise, set $x_{0}=-\min _{k, x_{k}<0} x_{k}$, and set $x_{j}^{\prime}=x_{j}+x_{0}$. Note that $x_{0}>0$ by construction. Thus, if for a particular $j, x_{j}>0$, then $x_{j}^{\prime}>0$. Suppose not. Then, $x_{j} \geq \min _{k, x_{k}<0} x_{k}$ and so $x_{j}^{\prime}=x_{j}-\min _{k, x_{k}<0} x_{k} \geq 0$. Thus, we have a solution $\mathbf{x}^{\prime}$ that satisfies (2).

\section{B Some Background on Determinants}

We review some useful results from the theory of determinants. All these results can be found in standard textbooks (e.g., [21, 24]).

Consider a $d \times d$ matrix $P$, where the $(i, j)$ th entry is denoted $p_{i, j}$ in the usual way. Then, the full product expansion of its determinant $|P|$ can be written as

$$
\sum_{\text {permutations } \pi \text { of }\{1,2, \ldots, d\}}(-1)^{\iota(\pi)} p_{1, \pi(1)} p_{2, \pi(2)} \ldots p_{d, \pi(d)}
$$

where $\iota(\pi)$ is the number of permutation inversions (swaps) in $\pi$.

The $(i, j)$ th minor of a matrix $P$ is the determinant of the submatrix obtained by deleting the $i$ th row and the $j$ th column of $P$. A minor of $P$ of order $r$ is the determinant of a square submatrix of $P$ of order $r$.

If we only fully expand $|P|$ along the first $k$ rows, we get the equation

$$
\sum_{\text {permutations } \pi \text { of }\{1,2, \ldots, k\}}(-1)^{\iota(\pi)} p_{1, \pi(1)} p_{2, \pi(2)} \ldots p_{k, \pi(k)} P_{k, \pi}
$$


where $P_{k, \pi}$ is the minor of $P$ obtained by excluding the first $k$ rows of $P$ and the $k$ columns corresponding to $\pi(1), \pi(2), \ldots, \pi(k)$.

The determinant of a matrix equals that of its transpose, i.e., $|P|=\left|P^{T}\right|$.

For an arbitrary $d \times d$ matrix $P$ with integral entries, we have the following bound on $|P|[7]$ :

$$
|P| \leq \frac{\mu_{P}^{d}(d+1)^{(d+1) / 2}}{2^{d}}
$$

where $\mu_{P}=\max _{(i, j)}\left|p_{i, j}\right|$. Equality is attained in certain cases.

A square, integer matrix $P$ is called unimodular (UM) if $|P| \in\{0,+1,-1\} . \quad P$ is called totally unimodular (TUM) if every square submatrix of $P$ is UM.

The node-arc incidence matrix of a directed graph is TUM. This matrix has entries in $\{0,+1,-1\}$ and every column has exactly one +1 entry and one -1 entry.

If $P$ is TUM, then so is $P^{T},[P \mid I]$, and $[P \mid-I]$. 